\title{
Constructed Invisibles: Designing Instruments as and for Effects
}

\author{
CATTY DAN ZHANG \\ University of North Carolina at Charlotte
}

Gabriele d' Annunzio described the "richest events occur in us long before the soul perceives them", and "when we begin to open our eyes to the visible, we have long since committed ourselves to the invisible". While unmediated human eye perceives the built environment from the visible forms, this paper discusses instruments and instrumentality through the development of a research project "Constructed Invisibles", which intends to build towards a volumetric perception of the environment with the awareness of the "void" in between. This project experiments ways of perceptually measuring the interaction between human behavior and unperceivable air movements; and explores in depth methods and techniques of designing with this invisible medium. Through tracking precedents in creating forms with airflow from principles of natural physics, this project seeks precision in articulating the invisible forms and patterns interactively following the geometric language. Points, lines, curves, surfaces, volumes and textures are generated through temperature and motion as agent.

"Constructed Invisibles" examines the medium, materiality, geometry and texture of airflow as material. It consists of a series of experimental devices with various strategies of utilizing thermal- and aerodynamics. Operations on the duration, interval, and electric current in controlling the thermal components, as well as on the speed, direction, and sequencing to manipulate the movements, allow invisible forms of the airflow being created, sensed, and choreographed. This project presents the integration of multiple systems, fabrication processes, optical experiments and computational analysis and design. It bears questions derived from processes and outcomes of the architectural and technological investigation on efficiency, effect, pragmatism, as well as on environmental aesthetics - with the potential of the invisible materials informing the design of the "visible".

"Air, gas, fire, sound, odors, magnetic forces, electricity,
electronics are materials. They must have two main func-
tions, namely: to protect against the rain, the wind and
atmospheric conditions in general and to create thermal
air conditioning." - Yves Klein, Lecture at Sorbonne in Paris, June 3, $1959^{1}$

In his interview by Laurent Stalder, Phillippe Rahm stated that: "Space form this point on was no longer imagined simply as void, as an absence defined by walls, floor and ceiling but as a less dense mass, disconnected, transparent and yet nevertheless filled with material; a void invisible to the eye, certainly, but in which the body was immersed." 2

As Rahm noted the importance of constructing atmosphere and climate within this void as fundamental role of architects, this invisible mass has been largely instrumentalized in contemporary practice of architecture. Although technological advancement such as machine vision, computational simulation, or imaging technologies allows us to sense and to design with air movements, thermal images, moisture, and so on in highly accurate ways, operations on these invisible elements support the visible forms and systems in aspects that are usually unperceived.

Arising out of the same attempt, another approach presents a shifted focus that's not on the scientific facts of the transparent medium, but on its perceptible performance as a fundamental material. This trend, for the past six decades, designed with principles of natural physics, manipulating ephemeral phenomenon for experiential effects. A vibrant, chaotic, and complex medium, air fills up the void. Airflow breaks and heals, taking on subtle forces and bringing huge impacts. Through a series of work researching on sculpting invisible forms, Constructed Invisibles presents a multi-stage development and extensive experiments. It explores the medium, materiality, geometry and texture of airflow, seeking ways to see and to articulate invisible forms based on geometric primitives.

This investigation assumes the visibility of the built environment as a volume - instead of a void space with surface boundaries - has impacts on our design cognition and on our body sensorium as an ensemble. A great amount of visuals with recurring patterns produced by distinct machines were captured and analyzed. In a highly scientific way, these instruments created and evolved during the process are integrated mechanisms and control systems, which are simultaneously artifacts themselves either blending or contrasting with the effects they generated and choreographed.

\section{MEDIUM}

Gabriele d' Annunzio elaborated the "richest events occur in us long before the soul perceives them", and "when we begin to open our eyes to the visible, we have long since committed ourselves to the invisible". Referencing the techniques of visualizing airflow, Schlieren Glasses- a wearable optical 
apparatus was developed as a beginning, in order to start to understand the richness of this invisible material intuitively by the action of seeing.

Flow visualization has long been explored in aeronautical engineering, combustion research, ballistics, and energy analysis. It is usually done through introducing tracers (dye, smoke or particles) into the fluid, and use particle image velocimetry (PIV) or optical flow algorithms to detect; or detecting the refraction of light rays through the fluid, where variations in index of refraction serve as tracers, using Schlieren photography and Schlieren PIV as ways for analyzing.

Perceptually measuring the interaction between human behavior and air movements, this apparatus utilizes a pair of scaled-down version of Schlieren imaging setups. Composed of small parabolic mirrors and LED light sources, it is precisely designed and assembled based on optical principles of making visible the light ray deviations (or wavefront deformations) due to the refractive index heterogeneities in a transparent medium. ${ }^{3}$ Designed to be a mobilized instrument that allows the visual impacts to interact with one's own movements, it measures two feet in length, ten inches wide, and a foot and half tall. With components interfacing with human body on the head and chest, one could wear it around as a pair of large glasses.
As the a number of light rays being bent before received by each eye, curvatures of airflow-originally generated by temperature difference and transformed by subtle movements - appear in a great range of forms constantly morphing at a high rate even if one is moving very slowly. (Figure 1 ).

\section{MATERIALITY}

Projects of utilizing airflow as material seemingly to fall within two categories: those aim to produce visible forms from pressure and tracer (fog, smoke, fabric, etc.), and those use only pressure to generate invisible air forms that one cannot experience while not "being there".

Art work on the matter of air boomed since Yves Klein's speculations in the 60s. Operations through sophisticated machine assemblies to provide pressure at various intensity, and direction from certain geometries of nuzzle, or arrangement of turbines, have presented typologies of forms that follow principles of natural physics. To design with airflow, fundamentally, is a process of choreographing systems that employ nature forces and effects.

Klein's interest in immaterial of spaces had led to his work in which air functioned as building structures and enclosures. Air Architecture, developed during 1957 -1962, envisioned a new social structure where solid walls and partitions were replaced by surfaces and furniture formed with compressed

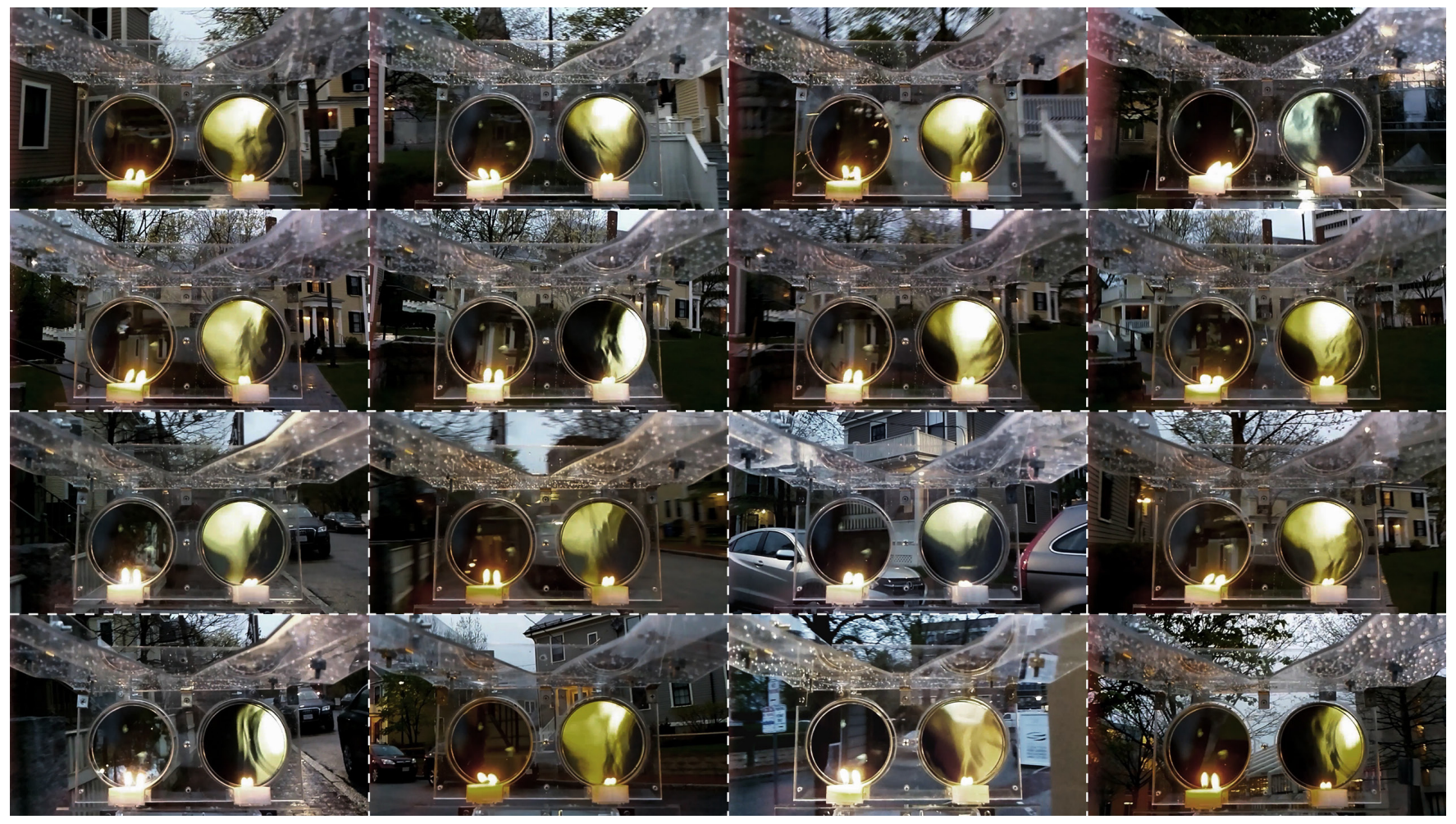

Figure 1: Frames extracted from video documentation of walking with Schlieren Glasses. Seeing airflow with naked eyes. 

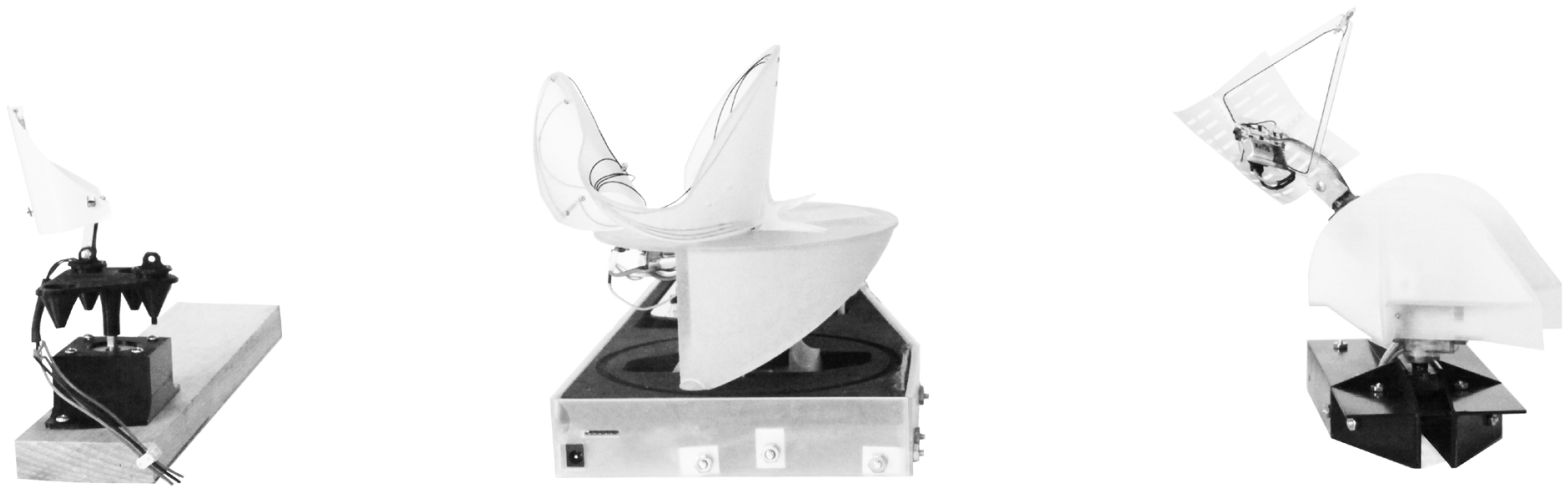

Figure 2: 3 prototypes for geometric study with heat source and motion control in various configurations.

air. The roof, a high-pressure injected air flow surface, shed water away and protected the habitat below.

This invisible barrier reconstructed social structure. As delineated by Klein:

"The air roof regulates the temperature and at the same time protects privileged area

There is a ground surface of transparent glass

There is an underground service area

The concept of secrecy, still common in our world, has disappeared in this city, which is flooded with light and completely open to the outside

A new atmosphere of human intimacy prevails." ${ }^{4}$

Michael Asher similarly utilized flow mechanics. His series of work titled Planes and Columns since 1966 was made from horizontal and vertical air currents. Vertical Column of Accelerated Air (1966-1967), stepped away from the functionality of a column - as apposed to the Air Roof by Klein. Instead, here it describes a pure volume of pressured air. Located between two adjacent spaces, the concealed fan in the ceiling generates a $40 \mathrm{~cm}$ thick column of downwards flow that creates a threshold as one enter from one side to the other, invoking bodily experiences through invisible sculptures.

The term "roof" or "column" here does not indicate the precise geometric properties of the forms generated. Instead, the transparency of air was maintained while a region within it-propelled by machinery-was altered to become certain pieces of architectural elements.

Natural typologies of forms of flow, on the other hand, could be reconstructed at certain capacity through aggregated or sequenced actions on manipulating pressure sources. Vortex, as one of the examples featured in Ned Kahn's installation Cloud Rings at 21-C Museum in 2006, is a ring of air that can travel large distances while keeping its shape and speed. A chamber of air with a flexible cover on one side and a small opening on the other, while taking a pressure input, the force coming from the flexible membrane travels through the chamber that filled with fog, exiting the small opening while forming the toroid vortex with the low pressure of air around. As the air accumulated at the opening, the puff of air eventually moves forward, becoming a ring.

Tornado, as another example, is usually derived in nature from a large volume/region of warm air that causes the reduced air pressure. To reconstruct this air turbulence artificially at various sizes and shapes generally requires an area of less air pressure, where air wants to accelerate to. In other words, a shear force generated by multiple air currents on one end (with fans, or warm rising air), and a negative force on the other is a typical controlled system.

\section{GEOMETRY}

The long process exploring the visibility and forms in this research undertakes fundamental questions: can we construct a comprehensive taxonomy of geometry with ephemeral and dynamic flow through an inclusive control system? Similar as constructing visible forms with any material, is there a precise way to articulate forms of airflow following geometric languages, from points to lines or curves; then to surfaces and volumes, so on so forth?

Observed from the wearable optical apparatus developed earlier, temperature and motion as agents are implemented in a series of designed instruments in order for forms to be generated. A point of heat in space, naturally presents an invisible straight line due to the up-flow current of warm air. Once this point starts to move, the straight line transforms into curves and dash lines, shaping by the forces from its surroundings. As other objects intervene, these curves would be sculpted into surfaces and volumes.

Navigated through electrical and mechanical systems, physics, and aerodynamic principles, these instruments consist 
of three main parts - heat points made from nichrome wire roughly 10-15ohm each; a motion system with a single or a combination of actuators providing certain degrees of freedom; and "fan blades" bringing in external forces. (Figure 2).

These machines are somehow drawing devices with constrained coordinates that specified for each iteration. Electrically calibrated for the sculpted forms being sensed by Schlieren optical apparatus, they require the control of a set of variables including current, resistance, as well as duration and interval for switching the heat on and off. Each of the devices is compactly designed and assembled that fits within the viewing area of a parabolic mirror $12.5^{\prime \prime}$ in diameter.

Experimented with various spatial configurations of "fan blades", arrangements of heat points and strategies of mapping two-dimensional heat gradient inputs to threedimensional patterns, machines are built, tested, and reassembled to resolve challenges encountered. These include the legibility in reading the depth datum from projected visuals captured through camera lenses, as well as heat disruptions produced from electronic components. A threeactuator system has been finalized for in-depth analysis. It accommodates the maximum capacity of motion trajectories for getting a wide range of constructed forms, and meanwhile maintaining the smallest amount of space the device itself occupies. Housed within a 3D-printed mechanism, each of the motors provides a 180 degree range of motion relative to the rotation of the others. Two points of heat situate on both sides of a perforated double-curved surface. One is near the convex center, while the other protrudes further on the other side.

Animated geometry sequences are modulated from scripted movements and temperature fluctuations. Velocity of the heat coils competes with the pace of the warm air rising, resulting in heat traces varying with the rhythm of motion along the same path. (Figure 3).

\section{TEXTURE}

The aesthetic experiments on creating and imaging the natural phenomenon differentiate themselves from drawing machines that precisely output digital signals to the desired position. As perfectly manifested in Henri Werle's photo Vortex Issued from a Rotor Blade Tip of a Helicopter in Forward Flight where dense vortices in specific order traced along a spiral trajectory, we see in these visual patterns a much wider vocabulary of flow. Lines that consist of clusters of points varying in density, or surfaces composed with jiggling curves which dissipate swiftly, add textural qualities to abstract shapes.

Elliptic vortices, buoyant jet, wake turbulence, etcfundamental features of the chaotic behavior of flow mechanics - are results of heterogeneous medium flowing at

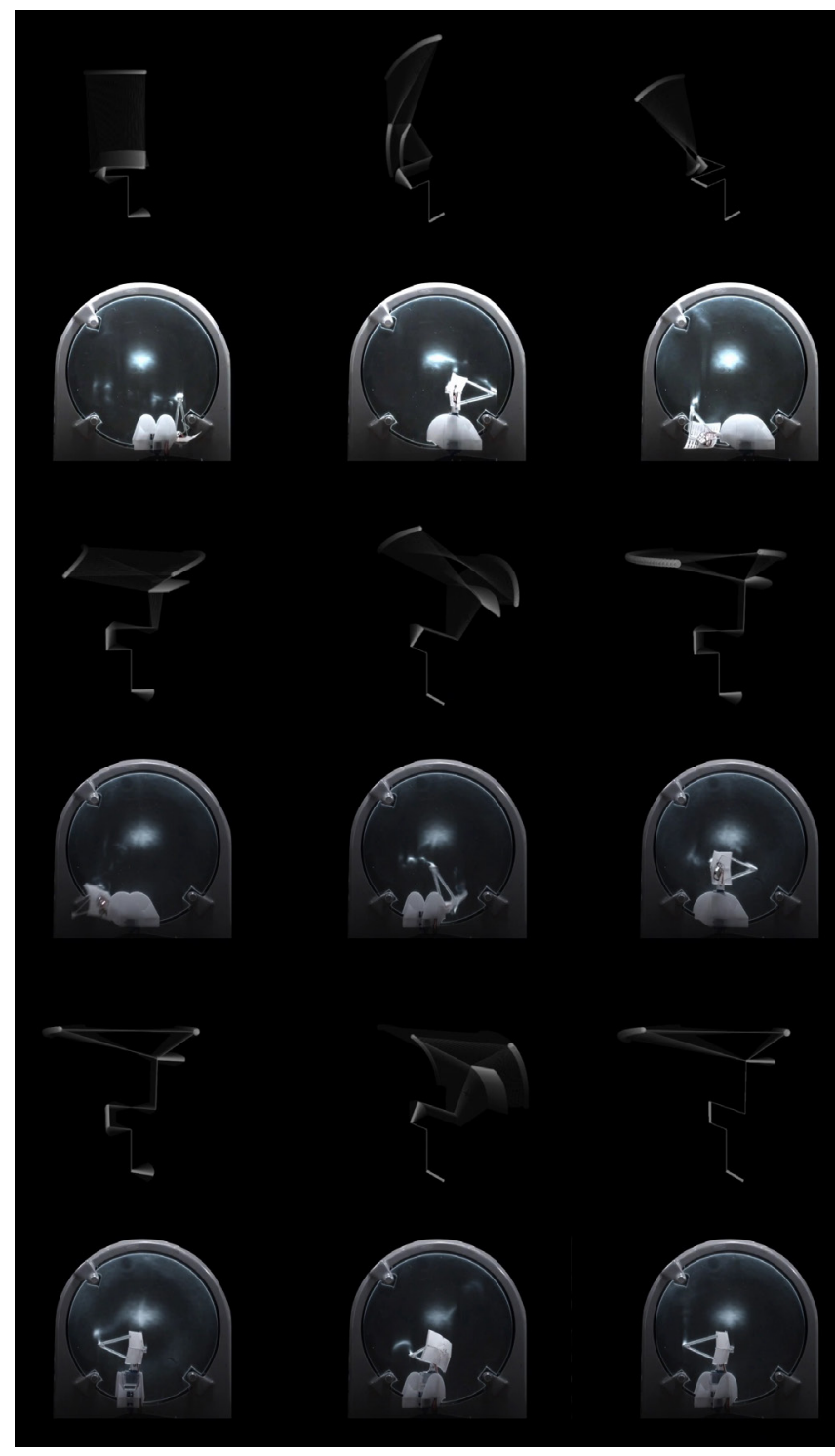

Figure 3: Invisible forms sculpted by designed machine with different scripted movements. Visualized by Schlieren Optics.

different speeds and directions. Within the same framework set up from the previous prototypes, a pair of cold elements is introduced for further investigation. Hanging from above, the down-flow air currents at times collide with the up-flow ones; while at other times affect the curvatures of the warm air from a distance.

The ability of controlling each of the temperature sources separately allows a great variety of motion sequences as well as temperature and spatial combinations being programmed. Each of the heat coils locates underneath an "umbrella", the center of which aligns with the mid-point of one edge. Situate towards the bottom of the viewing circle, two sets of skeleton holding the heat are driven by stepper motors that provide continuous rotary motion. Vertical sweep, as well as high frequency vibration of each umbrella, mimics the aerodynamic performance of insect wings (Figure 4). A hierarchy of traces 


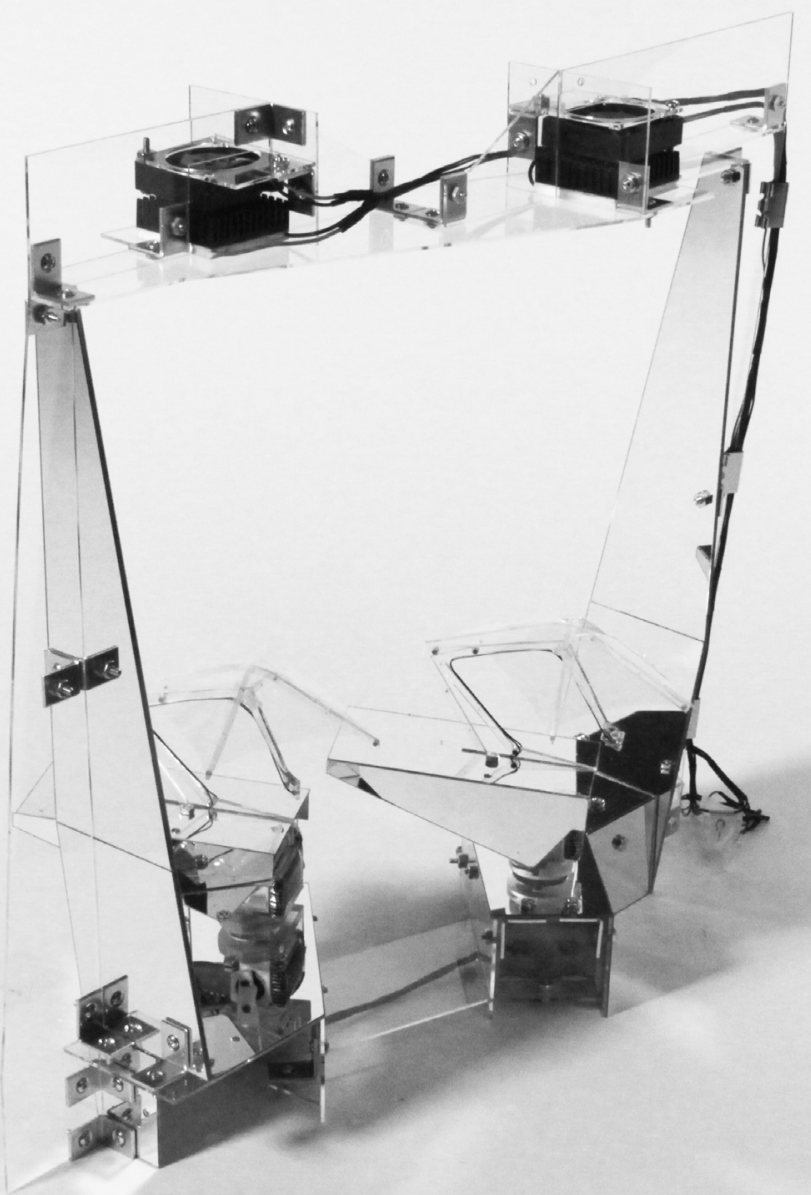

Figure 4: Multi-agent device with a pair of cold sources, a pair of heat coils, stepper motors, servo motors and DC motors for hierarchy of motion control.

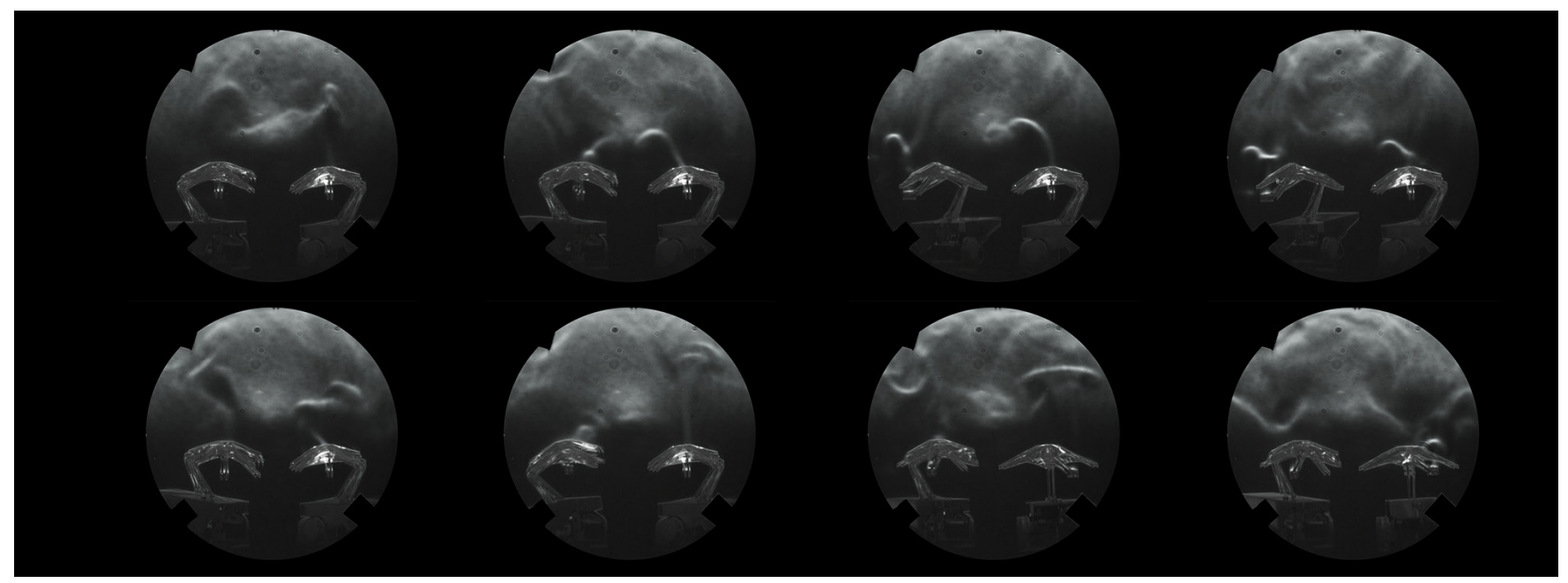

Figure 5: Invisible forms sculpted by designed machine with various scripted movements. Visualized by Schlieren Optics. 
with various levels of fuzziness is animated, interacting with the cold streams dropping at certain intervals. Symmetrical vortices rise; lines bend into vaults; wide plumes spread into thin curves... Mild motion inputs acts as stimuli, triggering rich dynamic patterns, evolving autonomously into formal narratives. (Figure 5).

\section{THE MACHINE? OR THE EFFECT?}

Steven Connor described that air was considered as next degree to nothing. As asserted in Robert Boyle's General History of the Air that "the generality of men are so accustomed to judge of things by their senses, that not finding the air to be a visible body, they ascribe less to it..." 5

Constructed Invisibles parallels design activities that are at the forefront of quantitative efficiency empowered by technological innovation. The availability of data that could not be directly comprehended by human are now drastically increased. This includes, but much more beyond, convection, air quality, meteorology, that directly or indirectly affects how we construct environmental systems and material assemblies. Constructed Invisibles, on the other hand, does not produce pragmatic uses. The digital instruments and designed machines synthesize into an indivisible whole, becoming intriguing by mediating its context of air forms.

This could trace back to what György Kepes argued in 1956 that "with instruments we are gradually finding a common denominator in all sensed experience; it is possible to convert sound to sight, space to time, light to form, and interchange phases and events, static and dynamic, sensible and conceptual". ${ }^{6}$ Kepes's vision on scientific instruments in relationship to art and sensation seventy years ago reflects profoundly the scientific experiments on these designed instruments in connection with Boyle's argument on visibility of air. By merging with the effects, these machines negotiate the balance in the design process where the invisible material perceptually informs the design of the visible.

Constructed Invisibles has not yet reached a conclusion and is far from being at an end. As an architectural investigation, it bears a wide range of different reactions and rigorous discussions among various groups of audiences. Some has struggles with science, technology and efficiency, while others celebrate the pure joy of visual and experiential impacts. The machines present ongoing opportunities to be evolved and scaled, yet the effects defy precise reproduction.

\section{ENDNOTES}

1 Francois Perrin, "Air Architecture: Imagination and Matter", Yves Klein: Air Architecture (Germany: Hatje Cantz Verlag, 2004).

2 Philippe Rahm: "Form and Function follow Climate", https://admin.arch.ethz. ch/vortragsreihe/pdf_archithese/Rahm_AR_2-10_s088-093.pdf

3 Michel Philbert, Jean Surget, and Claude Veret, "Shadowgraph and Schlieren," in Handbook of flow visualization, ed. Wen-Jei Yang (CRC Press, 2001), 195
4 Yves Klein - Werner Ruhnau, "Project of an Air Architecture," in Air architecture, 1951-2004, ed. Peter Noever and François Perrin (Ostfildern-Ruit : Hatje Cantz, c2004).

5 Steven Connor, The Matter of Air: Science and Art of the Ethereal (London, UK: Reaktion Books Ltd., 2010), 30.

6 György Kepes, The New Landscape in Art and Science (Chicago, IL: P. Theobald, 1956), 173. 